\title{
Modélisation régionale des débits de crue du bassin hydrographique du Cris : approche régionale classique et par modèles de référence
}

\section{Floods regional modeling of the Cris watershed : classical regional and reference models approach}

\author{
R. Mic, G. Galéa et P. Javelle
}

Volume 15, numéro 3, 2002

URI : https://id.erudit.org/iderudit/705475ar

DOI : https://doi.org/10.7202/705475ar

\section{Aller au sommaire du numéro}

\section{Éditeur(s)}

Université du Québec - INRS-Eau, Terre et Environnement (INRS-ETE)

\section{ISSN}

0992-7158 (imprimé)

1718-8598 (numérique)

\section{Découvrir la revue}

Citer cet article

Mic, R., Galéa, G. \& Javelle, P. (2002). Modélisation régionale des débits de crue du bassin hydrographique du Cris : approche régionale classique et par modèles de référence. Revue des sciences de l'eau / Journal of Water Science, 15(3), 677-700. https://doi.org/10.7202/705475ar

\section{Résumé de l'article}

Une régionalisation débit-durée-fréquence des débits de crue est réalisée sur les sous bassins du Cris qui draine une superficie d'environ $14300 \mathrm{~km}^{2}$ à l'ouest de la Roumanie. Cette régionalisation concerne 78 sous bassins dont les chroniques de débit quotidien et de pointe observées sont de trente ans en moyenne et pour lesquels nous disposons des pluies maximales de bassin de 1 jour à 10 jours calculées à partir de 92 postes pluviométriques. La régionalisation est menée selon deux approches : une approche régionale classique et une approche à partir de trois modèles adimensionnels de référence établis sur trois sites observés de France. La différence fondamentale entre les deux approches réside en ce que l'une prend en compte l'information spatiale pluie-débit inventoriée du Cris et que l'autre considère essentiellement l'information pluie-débit de chaque site de référence français. L'approche modèles de référence a pour base conceptuelle une typologie des crues qui pour un site cible est prédéfinie par un critère de choix, tandis que l'approche classique nécessite que soient définis des régions hydrologiques homogènes. Cette démarche est menée sur les trois sous bassins hydrographiques du Cris et permet d'étendre la région hydrologique homogène à l'ensemble du bassin du Cris. L'approche régionale comme l'approche modèles de référence privilégie la loi exponentielle adaptée aux valeurs supérieures à un seuil pour ce qui concerne les quantiles de crue de faible période de retour et pour des durées de $1 \mathrm{j}$ à $10 \mathrm{j}$ selon la dynamique de crue des sous bassins. Pour les quantiles de crue de grande période de retour les deux approches sous tendent le modèle du GRADEX, forme d'extrapolation des distributions observées par le gradex des pluies maximales. Quelle que soit l'approche de régionalisation, en tout site cible doivent être disponibles deux descripteurs de régime : le débit de pointe décennal Q10 et une durée caractéristique de crue D. Afin de comparer essentiellement l'incertitude des modélisations sur les quantiles de crue, D et Q10 sont connus et déduits des observations. Les résultats présentés montrent une bonne validité du modèle régional ajusté sur l'ensemble du Cris. Ceci indique que la zone étudiée est relativement bien homogène. Concernant les modèles de référence, leur critère de choix n'apparaît pas pertinent lorsqu'on s'intéresse aux faibles périodes de retour, mais se révèle significatif pour les fortes périodes de retour. Ce résultat est en grande partie dû à la méthode d'extrapolation appliquée. Celle ci est liée à la méthode du GRADEX et utilise l'information locale sur les gradex de pluie, comme cela est souvent le cas en France. Il est à noter que ces modèles de référence établis sur des chroniques de débit et pluie d'avant 1992 n'ont pas été réactualisés. L'exemple du bassin du Cris montre qu'ils n'en gardent pas moins un caractère opérationnel pour l'estimation des quantiles de crue de durée $\mathrm{d}(0<\mathrm{d}(\mathrm{j})<10)$ et de période moyenne de retour $\mathrm{T}(5<\mathrm{T}(\mathrm{an})<1000)$.
Ce document est protégé par la loi sur le droit d'auteur. L’utilisation des services d’Érudit (y compris la reproduction) est assujettie à sa politique d'utilisation que vous pouvez consulter en ligne.

https://apropos.erudit.org/fr/usagers/politique-dutilisation/ 


\title{
Modélisation régionale des débits de crue du bassin hydrographique du Cris : approche régionale classique et par modèles de référence
}

\author{
Floods regional modeling of the Cris watershed: \\ classical regional and reference models approach
}

R. MIC ${ }^{1}$, G. GALÉA ${ }^{2 *}$, P. JAVELLE ${ }^{3}$

Reçu le 20 décembre 2001, accepté le 6 juillet 2002 ${ }^{* *}$.

\section{SUMMARY}

When local information on streamflows is insufficient for estimating flood quantiles, a Regional Flood Frequency Analysis (RFFA) is usually carried out. Once homogeneous hydrological regions are defined, methods such as the "Index-Flood" method can be applied. In most cases, the variable understudy is the maximum peak flood (instantaneous or mean daily value, depending available data). However, the severity of a flood is not only defined by its peak, but also by its volume and duration.

For this reason, Cemagref has developed for several years an approach which, in addition to the classic flood/frequency relationship, takes into account the notion of duration: the flood-duration-frequency approach $(Q d F)$. In a similar manner to the rainfall intensity-duration-frequency analysis, averaged discharges are computed over different fixed durations $d$. For each duration, a frequency distribution of maximum averaged discharges is studied. Finally, a continuous formulation is fitted, as a function of the return period $(T)$ and the duration $(d)$ over which discharges have been averaged. Rare quantiles are determined using rainfall frequency information, according the so-called "GRADEX" method ("aesthetic" version). The regionalization of the QdF approach leaded to define three sets of dimensionless QdF curves. The originality of the method is that each set was fitted to one unique basin, located in France, and corresponding to a distinct hydrological regime.

1 INMH, 79 rue Bucuresti-Ploiesti, Sector 1 Bucarest, Roumanie.

2 Cemagref-Lyon, Unité de recherche hydrologie hydraulique, 3 bis quai Chauveau, 69336 Lyon cedex 09, France.

3 Centre for Water Resources Research, Civil Engineering Department, University College Dublin, Earlsfort Terrace, Dublin 2, Ireland.

* Correspondance. E-mail : gilles.galea@cemagref.fr

** Les commentaires seront reçus jusqu'au 30 avril 2003. 
A choice criterion, involving maximal rainfall distributions, determines which reference basin has to be considered. In this way, the QdF regionalization differs from the classic concepts mentioned in the first paragraph. The classic approach takes into account the whole streamflow-rainfall information available on a homogeneous hydrological region, while the QdF regionalization developed at Cemagref considers the streamflow-rainfall information available on the three French reference basins.

The aim of this research is to apply classical regional concepts (homogeneous regions) to the QdF approach and to compare results with thus obtained using the three French reference basins. One interest of the study is that it has been conducted in Romania, ie, outside the area where the three reference basins are located.

The case study is carried out on the Cris river sub-catchments which cover an area of about $14300 \mathrm{~km}^{2}$, in the west of Romania. This regionalization concerns 78 sub-catchments having about thirty years of streamflow measurements (daily flow and instantaneous flood peaks). For validation purpose, two basin sets are constituted: a calibration set ( 54 basins) and a validation set (24 basins). Furthermore, maximal rainfalls over 1 day to 10 days are available for these basins, from 92 rain-gauge stations.

First, regional QdF curves are deduced according the definition of homogeneous regions. Three regions are defined, corresponding to the three main sub-catchments of the Cris basins. After different tests, it is shown that the whole Cris basin can be considered as a unique homogeneous region. Then, the regionalization involving the three French reference basins is carried out. In both cases, methods are applied with the exponential law adjusted on peak over threshold values, for small return periods. Durations are ranging from 1 day to 10 days, according to the flood dynamic of the studied basins. For long return periods, both approaches use the GRADEX method, which extrapolates discharge distributions according to the rainfall distributions. Whatever the regional approach used, two descriptors have to be estimated for each target site, in order to unscale the dimensionless regional QdF curves. Theses two descriptors are the 10-year-return-period peak flood $Q 10$ and a flood characteristic duration $D$. In order to compare the uncertainty of the two approaches, $D$ and $Q 10$ are local values, deduced from observations.

The results presented shows a good validity of the regional model fitted to the whole Cris basin. This indicates that this region is quite homogeneous. Concerning the reference models, their choice criterion does not appear to be pertinent for small return periods, but becomes relevant for high return periods. In this case, estimations are comparable to thus provided using the whole regional information available on the Cris basin. This result is mainly due to the extrapolation method used. It is related to the GRADEX method and takes into account the local information about rainfall gradex, as it is often done in France. One point to be noticed is that these reference models have not been updated since they have been established in 1992. Despite that, the Cris basin example shows their operational ability for estimating flood quantiles of duration $d(0 \leq \mathrm{d}($ day $) \leq 10)$ and return period $T(5 \leq \mathrm{T}$ (year) $\leq 1000$ ). From an operational point of view, the three reference basins can be a valuable option. Indeed, a regional analysis is sometime difficult to carry out: data not available or too costly, time available for the study to short, etc. On the other hand, the three reference basins method is easier to apply and requires less data. However, uncertainties and hypothesis of the method should be kept in mind. In particular, we should be aware that high return period quantiles are given according the GRADEX method, ie using the frequency rainfall information. Consequently, the hypothesis of this method should be respected.

Key-words: flood flow, statistical hydrology, synthesis modeling, regionalization. 


\section{RÉSUMÉ}

Une régionalisation débit-durée-fréquence des débits de crue est réalisée sur les sous-bassins du Cris qui draine une superficie d'environ $14300 \mathrm{~km}^{2}$ à l'ouest de la Roumanie. Cette régionalisation concerne 78 sous-bassins dont les chroniques de débit quotidien et de pointe observées sont de trente ans en moyenne et pour lesquels nous disposons des pluies maximales de bassin de 1 jour à 10 jours calculées à partir de 92 postes pluviométriques. La régionalisation est menée selon deux approches : une approche régionale classique et une approche à partir de trois modèles adimensionnels de référence établis sur trois sites observés de France. La différence fondamentale entre les deux approches réside en ce que l'une prend en compte l'information spatiale pluie-débit inventoriée du Cris et que l'autre considère essentiellement l'information pluie-débit de chaque site de référence français. $L$ 'approche modèles de référence a pour base conceptuelle une typologie des crues qui pour un site cible est prédéfinie par un critère de choix, tandis que l'approche classique nécessite que soient définies des régions hydrologiques homogènes. Cette démarche est menée sur les trois sous-bassins hydrographiques du Cris et permet d'étendre la région hydrologique homogène à l'ensemble du bassin du Cris. L'approche régionale comme l'approche modèles de référence privilégie la loi exponentielle adaptée aux valeurs supérieures à un seuil pour ce qui concerne les quantiles de crue de faible période de retour et pour des durées de 1 jour à 10 jours selon la dynamique de crue des sousbassins. Pour les quantiles de crue de grande période de retour les deux approches sous tendent le modèle du GRADEX, forme d'extrapolation des distributions observées par le gradex des pluies maximales. Quelle que soit l'approche de régionalisation, en tout site cible doivent être disponibles deux descripteurs de régime : le débit de pointe décennal $Q 10$ et une durée caractéristique de crue $D$. Afin de comparer essentiellement l'incertitude des modélisations sur les quantiles de crue, $D$ et $Q 10$ sont connus et déduits des observations. Les résultats présentés montrent une bonne validité du modèle régional ajusté sur l'ensemble du Cris. Ceci indique que la zone étudiée est relativement bien homogène. Concernant les modèles de référence, leur critère de choix n'apparaît pas pertinent lorsqu'on s'intéresse aux faibles périodes de retour, mais se révèle significatif pour les fortes périodes de retour. Ce résultat est en grande partie dô à la méthode d'extrapolation appliquée. Celle ci est liée à la méthode du GRADEX et utilise l'information locale sur les gradex de pluie, comme cela est souvent le cas en France. Il est à noter que ces modèles de référence établis sur des chroniques de débit et pluie d'avant 1992 n'ont pas été réactualisés. L'exemple du bassin du Cris montre qu'ils n'en gardent pas moins un caractère opérationnel pour l'estimation des quantiles de crue de durée $d(0 \leq \mathrm{d}(\mathbf{j}) \leq 10)$ et de période moyenne de retour $T(5 \leq T($ an $) \leq 1000)$.

Mots clés : débit de crue, hydrologie statistique, modélisation de synthèse, régionalisation.

\section{1 - INTRODUCTION}

\subsection{Rappel sur la modélisation hydrologique statistique débit-durée-fréquence}

La bibliographie internationale recense de nombreux travaux qui traitent de la régionalisation des crues, ceux-ci privilégient la classique relation débit/fré- 
quence où le débit étudié est généralement le débit de pointe ou le débit journalier. Nous citerons par exemple ceux du Groupe de recherche en hydrologie statistique au Québec qui a fait un important travail de synthèse sur les différentes méthodes de régionalisation utilisées en Amérique du Nord (GREHYS, $1996 \mathrm{a}, \mathrm{b}$ ). De manière générale, une fois la région hydrologique homogène définie, la méthode dite de l'indice de crue (DALRYMPLE, 1960) est l'une des méthodes les plus couramment utilisées. Parallèlement à ces travaux le Cemagref a développé depuis plusieurs années une méthodologie qui associe aussi la notion de durée (PRUDHOMME, 1992-1995). Cette approche appelée débitdurée-fréquence (QdF) permet de prendre en compte la forme des crues et donc de donner une description statistique objective de leur variabilité temporelle. Il en a résulté 3 modèles adimensionnels dont la conceptualisation privilégie les types de crue observés en chacun des trois sites de référence plutôt que l'information régionale au sens des régions hydrologiques homogènes (GALÉA et PRUDHOMME, 1997a, b). Ces dernières années, JAVELLE (1997-2001) a consolidé et rationalisé le concept de modélisation (JAVELLE et al., 1999, 2000) qui permet, entre autres, de ne disposer que d'un seul modèle appelé modèle QdF convergent, en raison de la convergence observée des distributions de débit vers les faibles périodes de retour. Ce modèle est indépendant de la loi statistique choisie. Sa régionalisation est possible à partir d'une information hydrologique homogène et a été testée sur les débits de crue du bassin hydrographique de la Moselle française (GALÉA et al., 2001) ainsi qu'au Canada (JAVELLE et al., 2001).

Ce rappel historique nous a permis de situer le cadre de notre propos dont nous précisons maintenant l'objectif.

\subsection{Objectif}

II s'agit d'évaluer l'apport des trois modèles de référence (figure 1) appliqués au bassin du Cris en Roumanie (MIC, 1997-2001), comparativement à celui de modèles régionaux, c'est-à-dire calés sur l'ensemble de l'information hydrologique disponible dans cette région. Cette démarche trouve tout son sens en ingénierie où l'usage des modèles QdF de référence peut être un gain

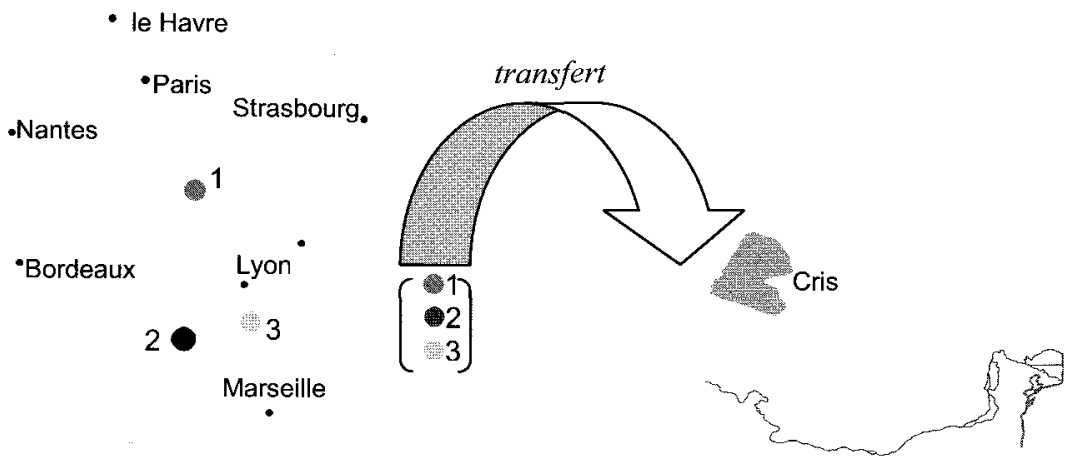

Figure 1 Modèles de référence : transfert des régimes de crue.

Reference models: flood regimes transfer. 
de temps important, comparativement à une démarche de régionalisation classique de mise en œuvre plus lourde et dont nous présenterons ultérieurement les principaux développements. La modélisation régionale a été menée à partir de la formulation associée aux modèles de référence. En d'autres termes, le modèle QdF convergent (JAVELLE et al., 1999) et sa régionalisation (GALÉA et al., 2001 ; JAVELLE et al., 2001) n'ont pas été appliqués ici. Ce choix nous est dicté par l'objectif que nous nous sommes fixés.

\section{2 - PRÉSENTATION DU RÉSEAU D'OBSERVATION HYDROPLUVIOMÉTRIQUE DU BASSIN DU CRIS}

L'information pluviométrique et hydrométrique retenue concerne respectivement 92 postes pluviométriques (figure 2) et 78 stations hydrométriques (figure 3). Le bassin hydrographique du Cris s'étend sur la Roumanie et la Hongrie et contrôle une superficie totale de $26600 \mathrm{~km}^{2}$. À l'ouest de la Roumanie sa superficie est d'environ $14300 \mathrm{~km}^{2}$. Elle est répartie en trois sous-bassins hydrographiques : le Crisul Alb (3911 km²), le Crisul Negru (4 $\left.495 \mathrm{~km}^{2}\right)$ et le Crisul Repede $\left(5878 \mathrm{~km}^{2}\right.$ ). Ces trois rivières donnent naissance au Cris qui se jette dans la rivière Tissa, affluent du Danube.

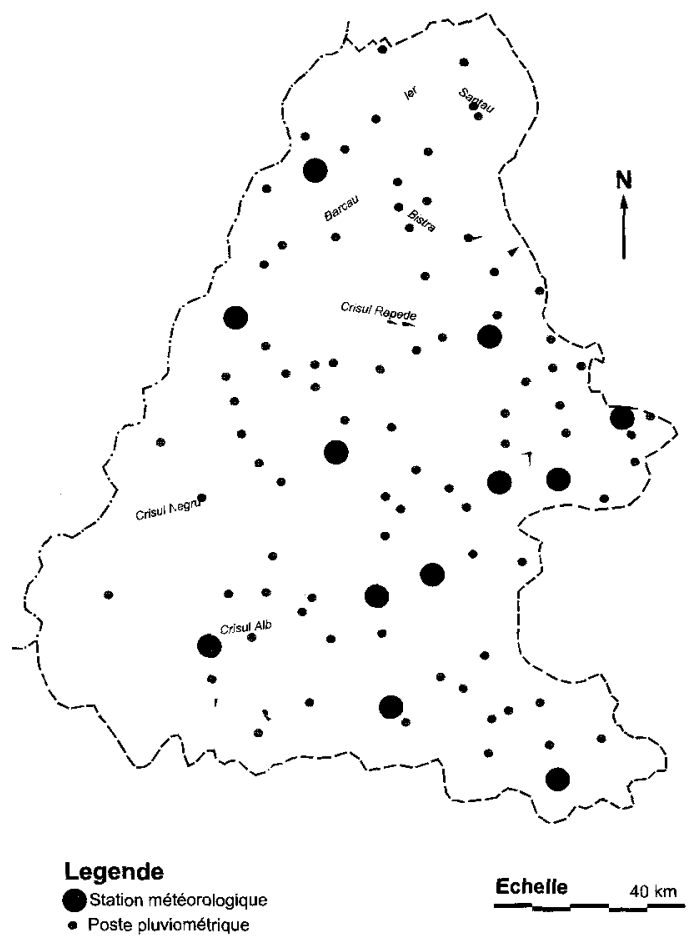

Figure 2 Présentation du réseau d'observation pluviométrique.

Rain gauge stations network. 


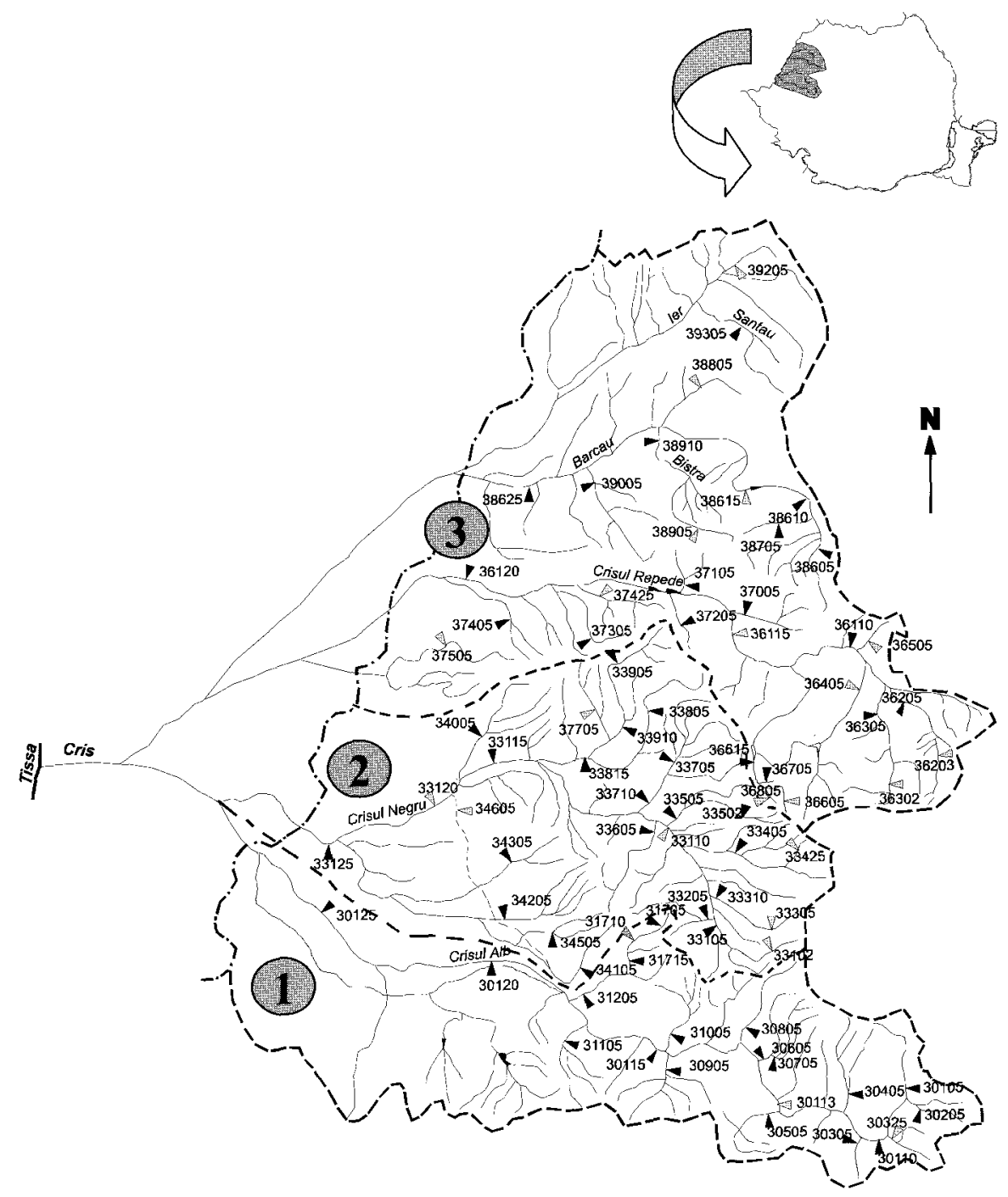

\section{Legende}

- Station hydrométrique de calage (54)

$40 \mathrm{~km}$

b Station hydrométrique de validation (24)

Figure 3 Situation du bassin hydrographique du Cris et des stations hydrométriques.

Cris catchment and streamflow gauge stations.

Parmi les 78 sous-bassins nous distinguerons, pour les besoins de la modélisation, un jeu de calage de 54 sous-bassins (18, par sous-bassin hydrographique) et un jeu de validation de 24 sous-bassins. Les classes de bassins étudiées sont les suivantes (figure 4). 

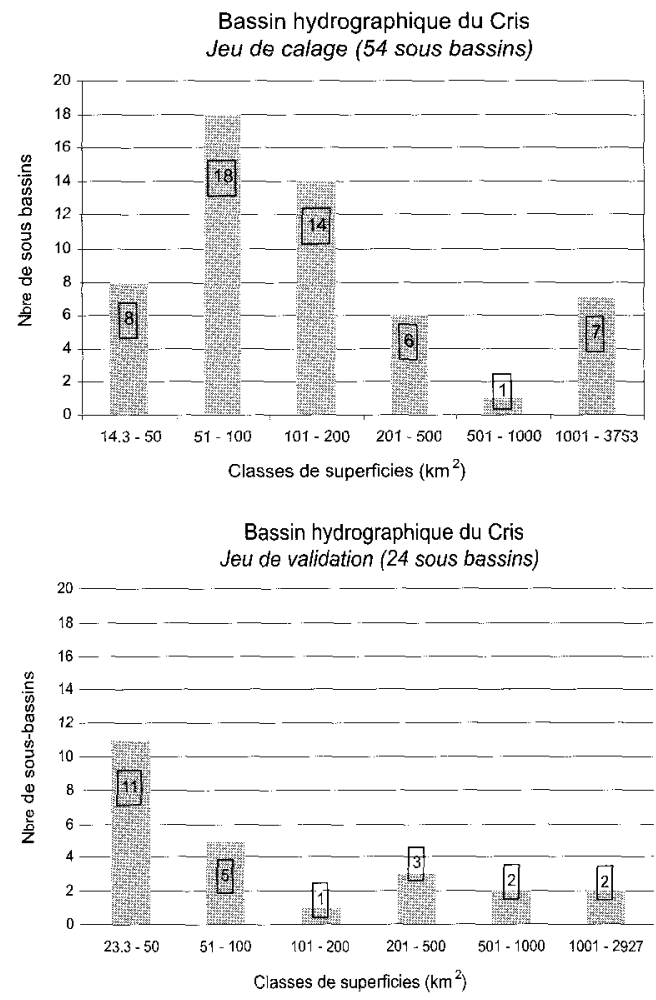

Figure 4 Classes de superficie des sous bassins retenus.

Range of selected catchments areas.

\section{3 - ANALYSE DES CRUES DE FAIBLES PÉRIODES \\ DE RETOUR $0,5 \leq T(A N) \leq 10$}

\subsection{Modélisation QdF locale}

\subsubsection{Définition des variables hydrologiques}

La variabilité temporelle des crues est étudiée à partir de deux variables hydrologiques (figure 5) traduisant deux notions de régime complémentaires : le débit moyen sur la durée continue $d$, maximal lors de la crue, noté VCXd et le débit seuil, continûment dépassé sur la durée $d$, maximal lors de la crue, noté $Q C X d$. Lorsque $\mathrm{d}=0$, les deux variables sont égales au débit instantané maximal $Q I X$.

Pour la suite, nous n'étudierons que la variable $V C X d$ et nous poserons par simplification $V \equiv V C X$.

\subsubsection{Ajustement statistique : Ioi exponentielle}

La loi de probabilité choisie, pour chaque échantillon de durée $d$ constitué, est une loi exponentielle adaptée aux valeurs supérieures à un seuil (COX, 

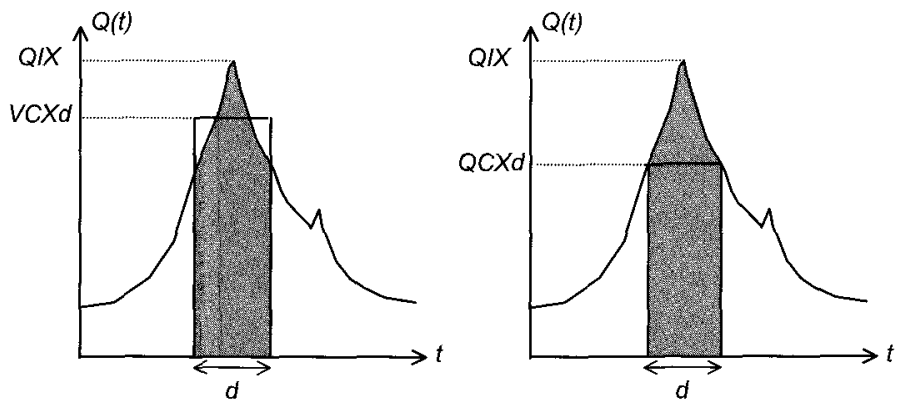

Figure 5 Définition des variables caractéristiques de crue.

Flood characteristic variables definition.

1962 ; FELLER, 1966) associées au processus de Poisson (LANG et al., 1999) et qui peut s'écrire (1) :

$$
V_{d}(T)=G q_{d} \cdot \ln (T)+Q O_{d}
$$

où $d$ en indice représente une valeur discrète de la durée ( $1 \mathrm{~s}$ ou $1 \mathrm{j}$, ou $3 \mathrm{j}$, etc.) ;

$T$ entre parenthèses signifie que la période moyenne de retour varie continûment ;

$G q_{d}$ : gradex des débits ou encore paramètre d'échelle de la loi ;

$\mathrm{QO}_{d}$ : paramètre de position de la loi ;

$T(\mathrm{an})=n a / n s\left(1-F_{i}\right)$;

na : le nombre d'années d'observation, $n s$ : nombre de valeurs ;

avec $F_{i}=\frac{i-0,3}{n s+0,4}$ fréquence au non dépassement $(i$ : rang) ;

$G q_{d}=m-V_{i=1} \quad$ ( $m$ : moyenne des débits $; V_{i=1}$ : le plus petit des débits) ; $Q O_{d}=V_{i=1}+G q_{d \cdot} / n(n s / n a)$.

Les deux paramètres, $G q_{d}$ et $Q 0_{d}$ une fois définis, permettent de tracer dans un repère approprié (figure 6 ) la courbe théorique $V_{d}(T)$ en fonction de $\ln (T)$.

\subsubsection{Passage des distributions $V_{d}(T)$ au modèle continu local $V_{L}(d, T)$}

Cette modélisation locale va essentiellement concerner le passage des quantiles de débit $V_{d}(T)$ donnés par la relation (1) aux quantiles consolidés avec la durée $V_{L}(d, T)$.

Pour cela, les paramètres des fonctions $G q(d)=\frac{1}{x 1 \cdot d+x 2}+x 3$ et $Q O(d)=\frac{1}{x 4 \cdot d+x 5}+x 6$ sont calés par optimisation, d'où la relation (2).

$$
V_{L}(d, T)=\left[\frac{1}{x 1 \cdot d+x 2}+x 3\right] \cdot \ln (T)+\left[\frac{1}{x 4 \cdot d+x 5}+x 6\right]
$$

Nous donnons une évaluation de l'adéquation de la modélisation à partir de l'erreur quadratique moyenne définie selon (3). 


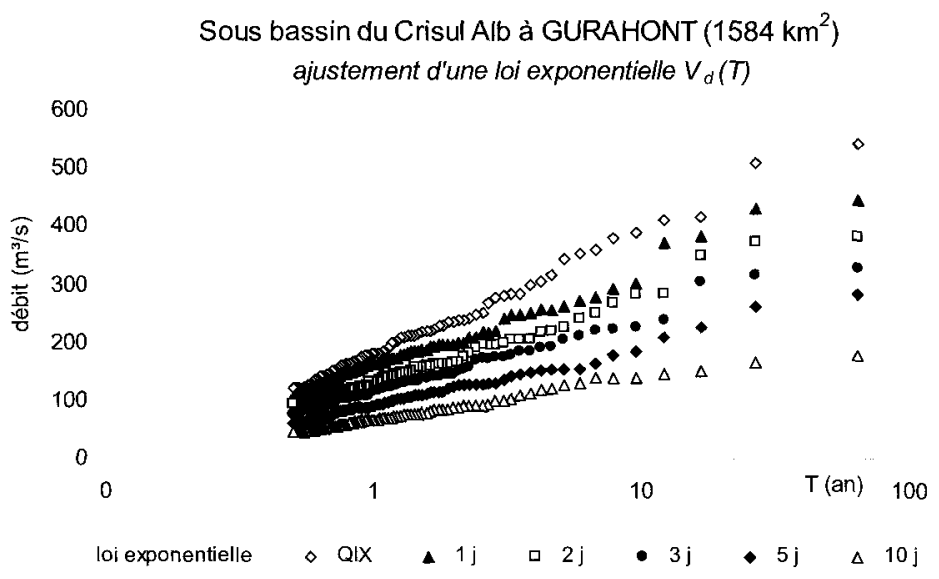

Figure 6 Loi exponentielle ajustée sur les échantillons de débit (code station : 30115).

Exponential distribution fitted to the discharge samples (station code: 30115).

Le nombre $n$ de durées $d$ considérées n'étant pas le même pour chacun des 54 sous-bassins $(n b=54)$ du fait de leur dynamique de crue différente, nous écrirons que :

$$
E r_{\text {quantile }}=100 \cdot \sqrt{\frac{1}{n b} \sum_{k=1}^{n b} \frac{1}{n} \sum_{i=1}^{n}\left(\frac{V_{k_{d_{i}}}(T)-V_{k_{L}}\left(d_{i}, T\right)}{V_{k_{d_{i}}}(T)}\right)^{2}}
$$

avec $E r_{\text {quantile }}$ : erreur quadratique moyenne (\%).

Nous donnons pour exemple à la figure 7 une représentation de cette erreur (3). De manière générale, l'incertitude (3) des modélisations QdF locales (2) sur les quantiles de crue observés (1) est faible.

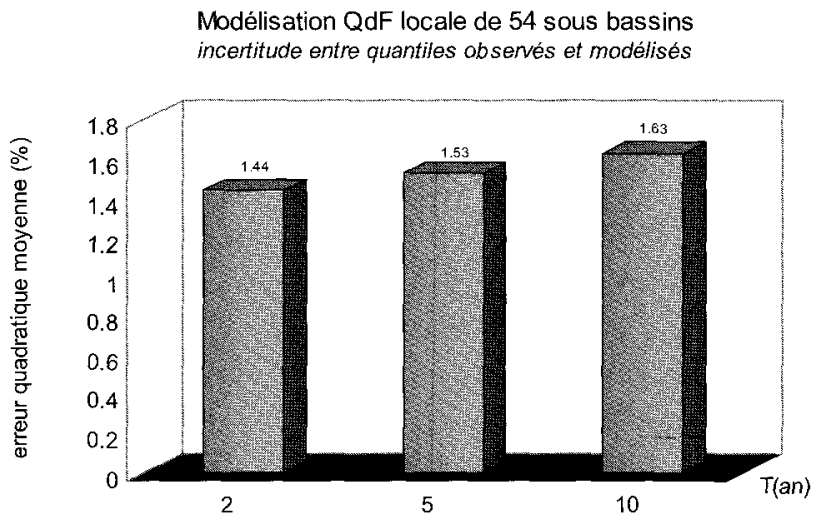

Figure 7 Incertitude liée à la modélisation locale (54 sous-bassins).

Local modeling (54 sub-catchments). 


\subsection{Approche régionale classique}

\subsubsection{Phase de calage}

Pour chacun des 3 sous-bassins hydrographiques nous considérons un jeu de calage de 18 sous-bassins, ce qui correspond à un jeu de calage de 54 sous-bassins pour le bassin hydrographique du Cris.

Dès à présent, nous considérons que les quantiles déduits de la modélisation locale (2) représentent l'information consolidée et homogénéisée des observations de débit échantillonnées pour des valeurs discrètes de $d$ (1).

Pour chacun des 54 sous-bassins on effectue la transformation (4) de Gq(d) et de QO(d) ainsi que le calage des paramètres Xi correspondants.

$$
\begin{aligned}
& G q(d / D) / Q 10=\frac{1}{X 1 \cdot d / D+X 2}+X 3 \\
& Q O(d / D) / Q 10=\frac{1}{X 4 \cdot d / D+X 5}+X 6
\end{aligned}
$$

où Q10 : débit de pointe décennal est déduit de (2): $Q 10 \cong V_{L}(0,10)$;

$D$ : durée caractéristique de crue du sous-bassin (CTGREF, 1980).

Pour le Crisul Alb à Gurahont le graphe de la figure 8 présente les couples (ds, $Q I X)$ des crues sélectionnées, ds correspond au temps où $Q I X / 2$ est dépassé. Le temps médian des plus fortes crues au voisinage de Q10 permet une estimation de $D$ proche de $40 \mathrm{~h}$.

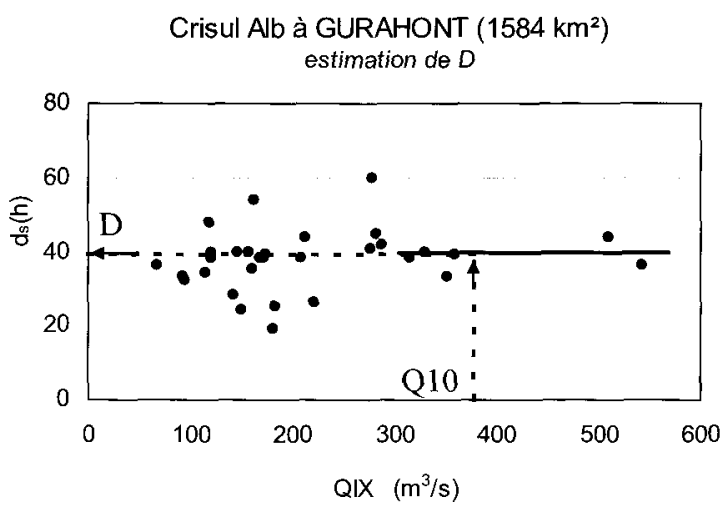

Figure 8 Estimation de $D$.

D estimation.

La figure 9 présente les courbes locales des gradex $G q(d / D) / Q 10$ et des paramètres de positions $Q 0(d / D) / Q 10$ (relation 4) obtenues pour 18 sous-bassins du Crisul Negru (région 2 sur la carte figure 3). À partir de ces faisceaux de courbes sont déduites des courbes régionales adimensionnelles, respectivement notées $G q_{R}(d / D)$ et $Q 0_{R}(d / D)$. De même qu'en (4), ces courbes sont définies par une forme hyperbolique à trois paramètres, respectivement $X 1_{R}, X 2_{R}$, $X 3_{R}$ et $X 4_{R}, X 5_{R}, X 6_{R}$. Chaque paramètre $X i_{R}$ est calculé en effectuant la 
moyenne des paramètres locaux $X i$ au sein de la région hydrologique considérée (DALRYMPLE, 1960). Pour le sous bassin hydrographique du Crisul Negru, les courbes régionales apparaissent en trait foncé sur les graphiques de la figure 9 .
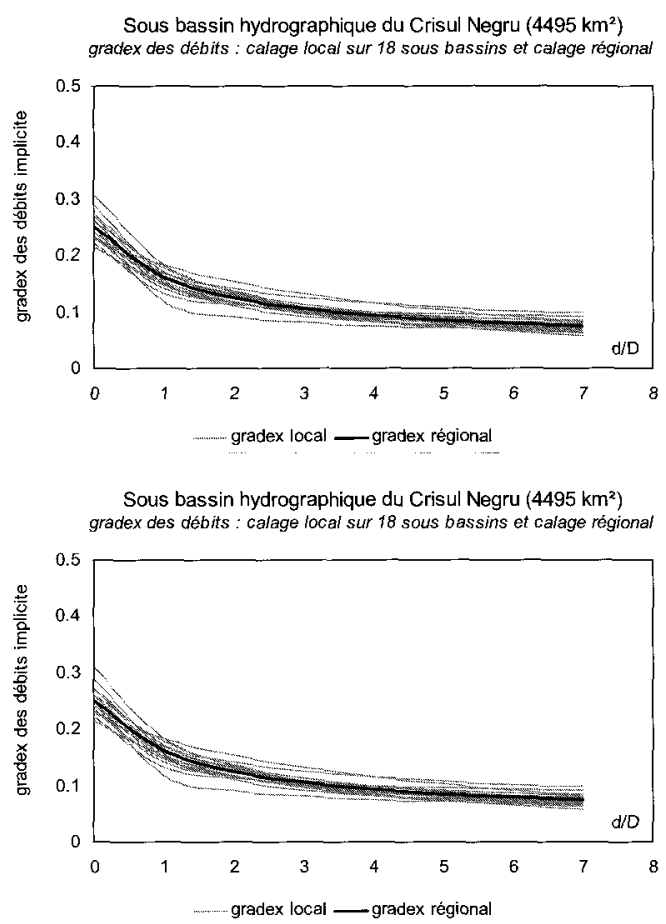

Figure 9 Gradex des débits et paramètre de position local et régional (Crisul Negru).

Local and regional discharges gradex and position parameters.

De la même façon, les courbes régionales adimensionnelles $G q_{R}(d / D)$ et $\mathrm{QO}_{R}(d / D)$ ont été calculées pour les deux autres régions considérées (figure 3 ) : les sous-bassins du Crisu Alb (région 1) et ceux du Crisu Repede (région 3). De plus, ces courbes ont aussi été calculées pour l'ensemble du bassin du Cris, c'est-à-dire en regroupant les trois regions étudiées. Les differents paramètres régionaux obtenus figurent au tableau 1 .

Le calcul des quantiles de crue au site cible se fera à partir de l'équation (5), connaissant les descripteurs de régime du site cible : $Q 10_{\text {site cible }}$ et $D_{\text {site cible }}$. Les quantiles de crue, en un site cible, déduits de la modélisation régionale seront notés $V_{R}(d, T)$.

$$
\begin{gathered}
V_{R}(d, T)_{\text {site cible }}=\left[\left(\frac{1}{X 1_{R} \cdot d / D_{\text {site cible }}+X 2_{R}}+X 3_{R}\right) \cdot \ln (T)+\right. \\
\left.\left(\frac{1}{X 4_{R} \cdot d / D_{\text {site cible }}+X 5_{R}}+X 6_{R}\right)\right] \cdot Q 10_{\text {site cible }}
\end{gathered}
$$


Tableau 1 Valeurs des paramètres régionaux relatifs à chaque entité hydrographique.

Table 1 Regional parameters value for each hydrological region.

\begin{tabular}{|c|c|c|c|c|c|c|}
\hline Sous Bassins Hydrographiques & $X 1_{R}$ & $X 2_{R}$ & $x_{R}$ & $X 4_{R}$ & $X 5_{R}$ & $x_{6}$ \\
\hline Crisul Alb (3911 km²) & 3,221 & 5,014 & 0,040 & 1,368 & 2,851 & 0,084 \\
\hline Crisul Negru (4 $\left.495 \mathrm{~km}^{2}\right)$ & 3,418 & 4,749 & 0,040 & 1,386 & 2,822 & 0,074 \\
\hline Crisul Repede (5 $878 \mathrm{~km}^{2}$ ) & 3,746 & 4,711 & 0,045 & 1,518 & 3,022 & 0,080 \\
\hline Bassin Hydrographique total & $X 1_{R}$ & $X 2_{k}$ & $X 3_{R}$ & $X 4_{R}$ & $x 5_{R}$ & $x 6_{R}$ \\
\hline Cris $\left(14300 \mathrm{~km}^{2}\right)$ & 3,468 & 4,824 & 0,042 & 1,421 & 2,896 & 0,079 \\
\hline
\end{tabular}

Les résultats du tableau 1 portés sur un même graphe (figure 10) montrent que la région hydrologique homogène peut être simplement représentée par la région géographique relative au bassin hydrographique du Cris.

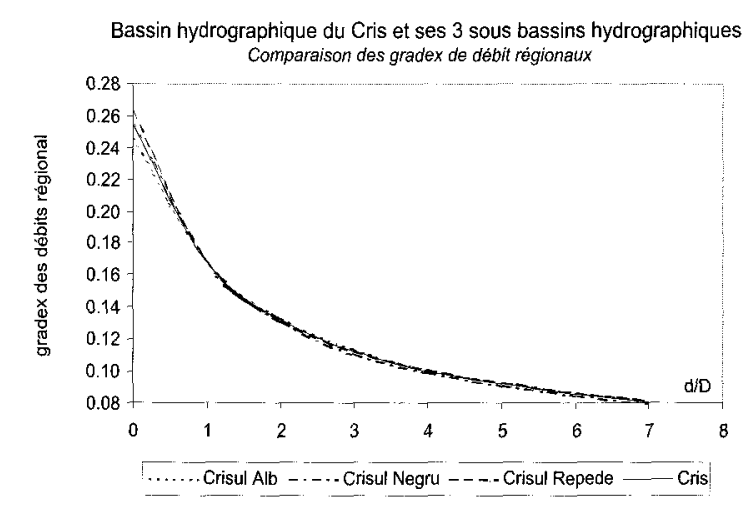

Bassin hydrographique du Cris et ses 3 sous bassins hydrographiques Comparaison des paramétres de position régionaux

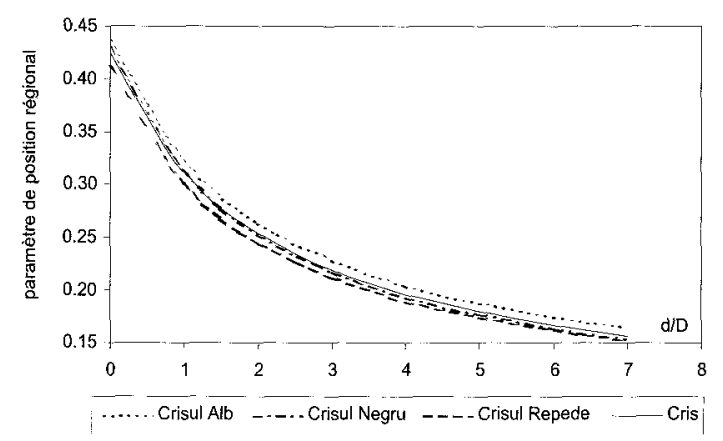

Figure 10 Comparaison des paramètres régionaux.

Regional parameters comparison.

L'équation (5) permet le calcul des quantiles de crue de durées $d$ compatibles avec la dynamique de crue des sous-bassins $(0 \leq \mathrm{d}(j) \leq 10)$ et pour des périodes moyennes de retour $T$ comprises entre deux fois par an et 20 ans 
$(0,5 \leq \mathrm{T}(\mathrm{an}) \leq 20)$. Vingt ans est une limite supérieure acceptable de la validité de (5) qui est de 10 ans, seuil d'extrapolation (cf. ultérieurement) aux grandes périodes de retour du modèle QdF régional du Cris (5).

Pour tester la robustesse du modèle QdF régional du Cris (5) par rapport à la modélisation QdF locale (2), nous nous mettons toujours dans le cas où Q10 (2) et $D$ sont connus.

Pour le jeu de 54 sous-bassins les figures 11 et 12 présentent trois graphes de comparaison pour un même quantile modélisé ainsi qu'un graphe de synthèse de l'erreur selon (6).

$$
E r_{\text {quantile }}=100 \cdot \sqrt{\frac{1}{n b} \sum_{k=1}^{n b} \frac{1}{n} \sum_{i=1}^{n}\left(\frac{V_{k_{L}}\left(d_{i}, T\right)-V_{k_{R}}\left(d_{i}, T\right)}{V_{k_{L}}\left(d_{i}, T\right)}\right)^{2}}
$$

L'erreur induite par la modélisation régionale (figure 12) par rapport aux modélisations locales reste acceptable pour le jeu de calage de 54 sous-bassins.

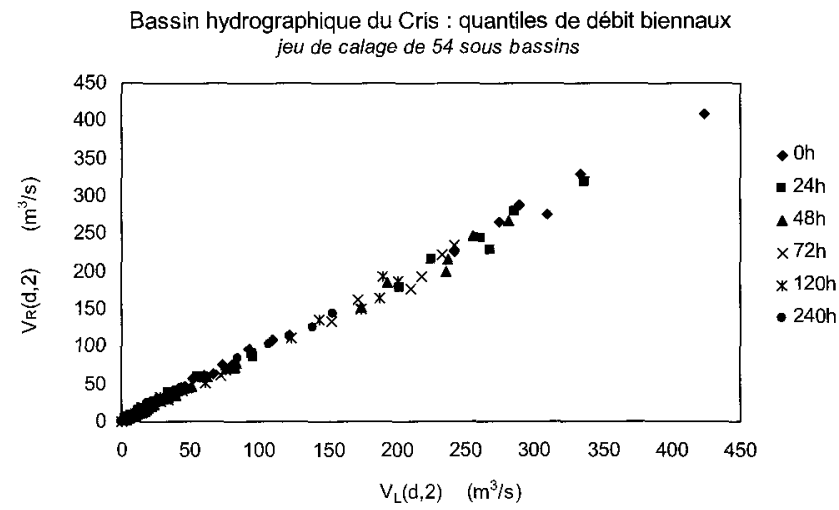

Bassin hydrographique du Cris : quantiles de débit quinquennaux jeu de calage de 54 sous bassins

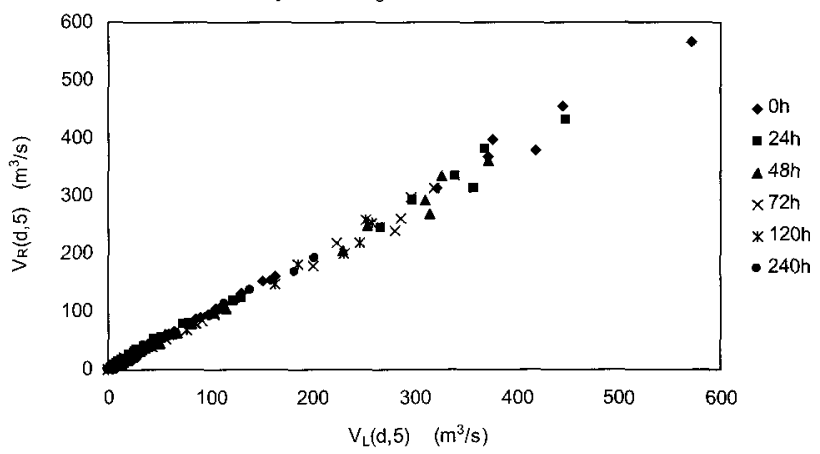

Figure 11 Modélisation locale (2) et régionale (5), comparaison des quantiles 2 ans et 5 ans.

Local (2) and regional (5) modeling, 2-year and 5-year quantiles comparison. 


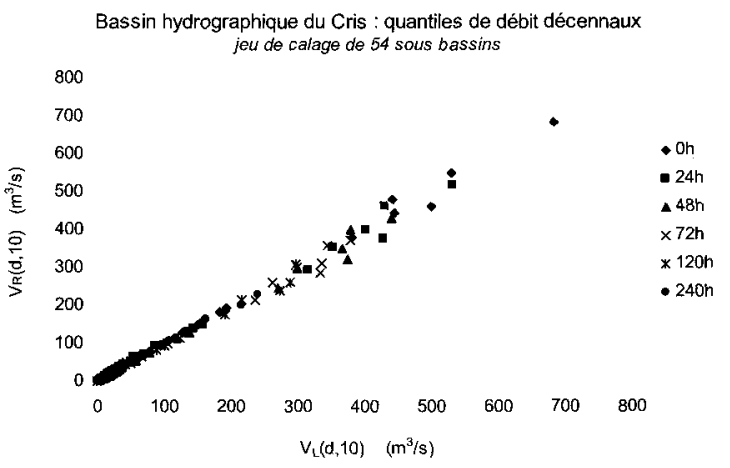

Modélisation QdF locale et régionale incertitude entre quantiles modélisés, jeu de calage de 54 sous bassins

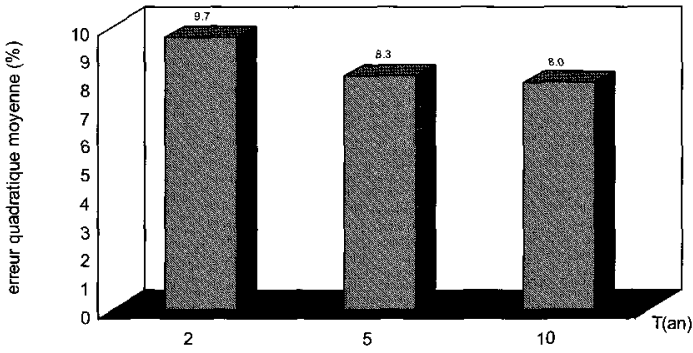

Figure 12 Modélisation locale (2) et régionale (5), comparaison des quantiles 10 ans et graphe de synthèse.

Local (2) and regional (5) modeling, 10-year quantiles comparison and synthesis graph.

\subsubsection{Phase de validation}

Elle concerne un jeu de 24 sous-bassins (figure 3) répartis sur l'ensemble des sous-bassins hydrographiques du Cris.

De même, les figures 13 et 14 permettent de confirmer la validation du modèle QdF régional du Cris (5) pour le jeu de 24 sous-bassins retenus. Le graphe de synthèse $(n b=24)$ de l'erreur selon $(6)$ montre que celle ci est d'un ordre de grandeur comparable à celle du jeu de calage.

\subsection{Approche par modèles QdF de référence}

L'approche par modèles de référence est ici brièvement rappelée. Pour plus d'information, on pourra se reporter à GALÉA et PRUDHOMME (1997a) et JAVELLE et al. (2000).

Les modèles de référence sont du même type que (5) où $R$ sera substitué par ref. La principale différence avec l'approche précédemment présentée est qu'ils ont été établis sur l'observation pluie débit d'un seul bassin (figure 1). Les pluies, comme nous le verrons ultérieurement, sont utilisées pour l'estimation des quantiles de crue de grande période de retour. Le jeu de paramètres adéquat (tableau 2) dépend d'un critère de choix du modèle de référence. Ce cri- 

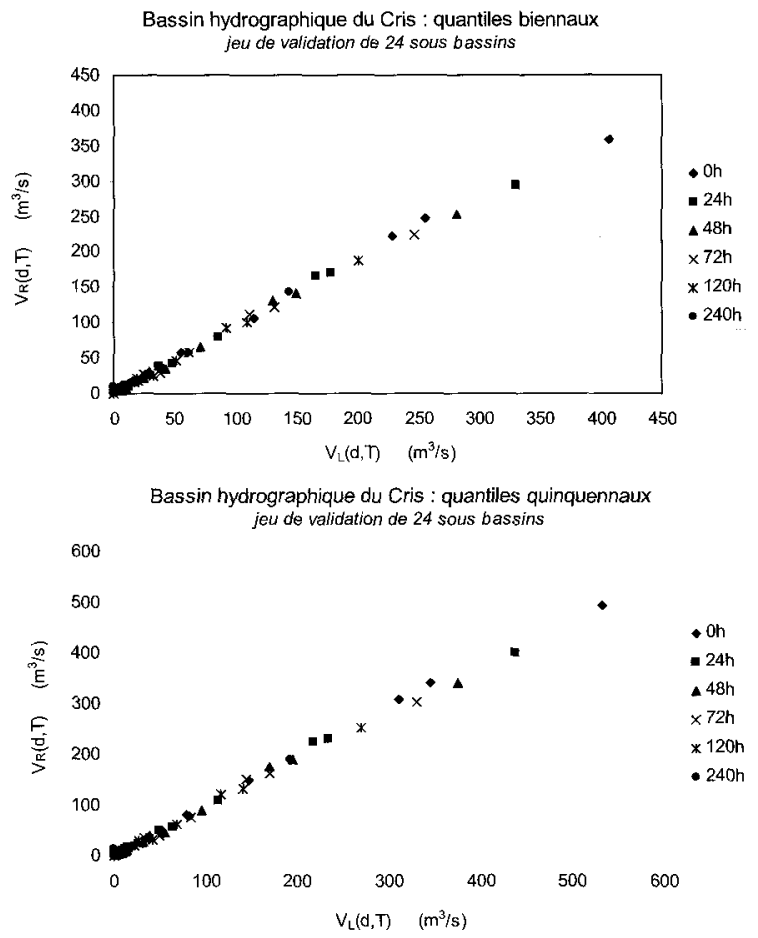

Figure 13 Validation du modèle régional, comparaison des quantiles 2 ans et 5 ans.

Regional model validation, 2-year and 5-year quantiles comparison.

Tableau 2 Valeurs des paramètres des trois modèles QdF de référence.

Table 2 Parameters value of the three reference models.

\begin{tabular}{|c|c|c|c|c|c|c|}
\hline $\begin{array}{c}\text { Modèles QdF } \\
\text { de référence }\end{array}$ & $\mathbf{X}_{\text {ref }_{\text {ref }}}$ & $\mathbf{X 2}_{\text {ref }}$ & $\mathbf{X 3}_{\text {ref }}$ & $\mathbf{X}_{\text {ref }}$ & $\mathbf{X}_{\text {ref }}$ & $\mathbf{X 6}_{\text {ref }}$ \\
\hline$(1)$ & 2,635 & 6,190 & 0,016 & 1,045 & 2,385 & 0,172 \\
$(2)$ & 1,120 & 3,560 & 0,000 & 0,950 & 3,180 & 0,039 \\
$(3$ & 0,870 & 4,600 & 0,000 & 1,070 & 2,500 & 0,099 \\
\hline
\end{tabular}

tère consiste à représenter pour différentes durées les gradex de pluie $G p_{d}$ du bassin étudié (exprimé en $\mathrm{m}^{3} / \mathrm{s}$ à l'aide de la surface du bassin), normés par le débit décennal $Q 10$, en fonction de $d / D$ (Q10 et $D$ étant relatifs au bassin étudié). Puis, le choix du modèle de référence s'effectue en fonction de la position des ratios $G p_{d} / Q 10$ sur le graphe de la figure 15. Les limites (1)/(2) et (2)/(3) ont été définies expérimentalement par PRUDHOMME (1992-1995).

Le choix du modèle de référence peut être effectué pour les 24 sous-bassins du jeu de validation pour lesquels nous disposons des gradex des plus fortes pluies de bassin de 1 jour à 10 jours (MIC, 1997-2001); les descripteurs de régime $Q 10$ et $D$ étant par ailleurs connus. 


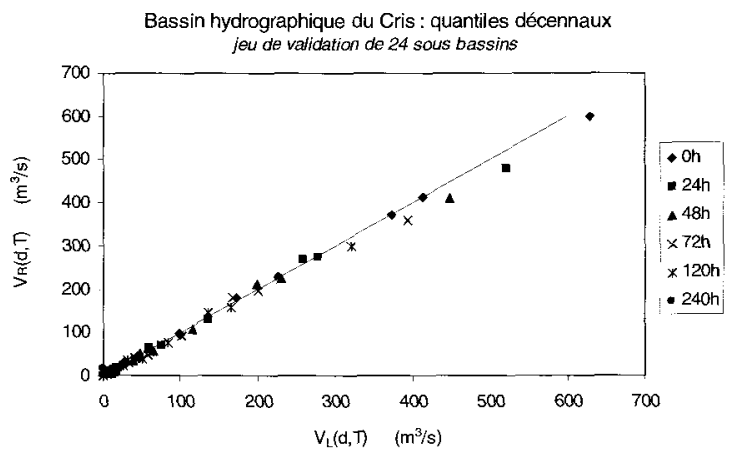

Modélisation $\mathrm{QdF}$ locale et régionale incertitude entre quantiles modélisés, jeu de validation de 24 sous bassins

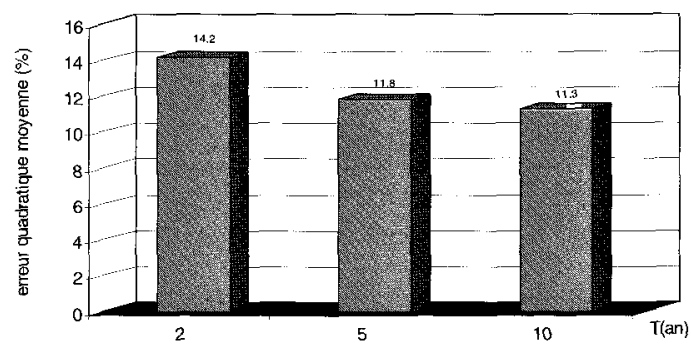

Figure 14 Validation du modèle régional, comparaison des quantiles 10 ans et graphe de synthèse.

Regional model validation, 10-year quantiles comparison and synthesis graph.

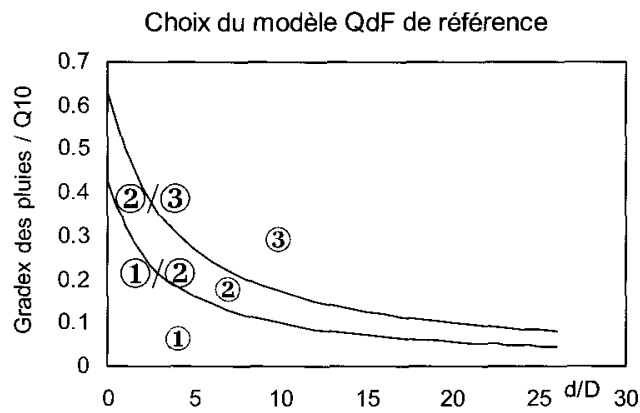

Figure 15 Définition du critère de choix.

Choice criterion definition.

Pour vérifier l'objectivité de ce choix, pour les quantiles de faible période de retour, nous avons par ailleurs choisi le modèle de référence (le meilleur) donnant la plus petite erreur quadratique moyenne selon (7). 


$$
E r_{\text {quantle }}=100 \cdot \sqrt{\frac{1}{n b} \sum_{k=1}^{n b} \frac{1}{n} \sum_{i=1}^{n}\left(\frac{V_{k_{L}}\left(d_{i}, T\right)-V_{k_{\text {ted }}}\left(d_{i}, T\right)}{V_{k_{L}}\left(d_{i}, T\right)}\right)^{2}}
$$

où $V_{\text {Kref }}\left(d_{i}, T_{\text {site cible }}\right.$ : quantile déduit du modèle $\mathrm{QdF}$ de référence.

La figure 16 permet de présenter les différents résultats obtenus.

Modélisation $Q d F$ régionale et de référence

incertitude liée à la modélisation, jeu de validation de 24 sous bassins

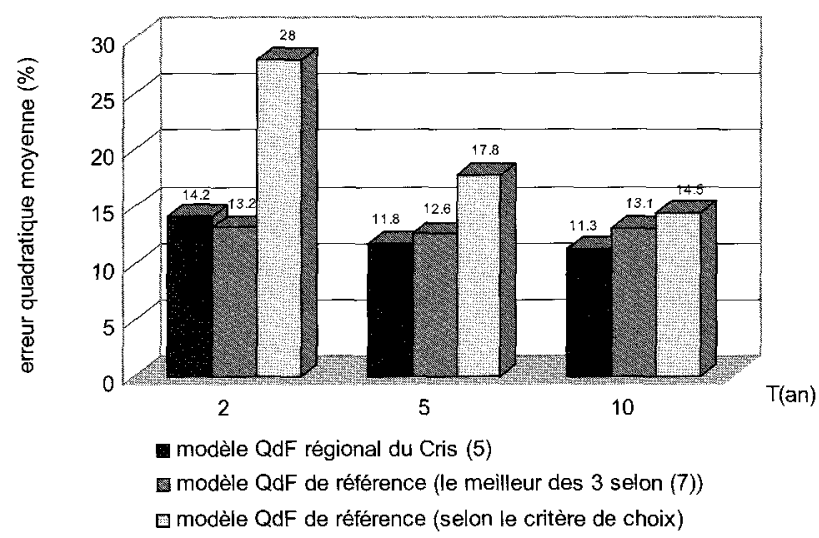

Figure 16 Comparaison des erreurs quadratiques moyennes des modèles $Q d F$. Comparison of $Q d F$ models quadratic errors.

Comme l'avaient déjà souligné les travaux de JAVELLE (1997-2001) sur le bassin hydrographique de la Moselle, le critère de choix (PRUDHOMME, 19921995) est en général peu pertinent pour les quantiles de faible période de retour. II faut cependant noter ici le caractère tranché du test par rapport à celui effectué pour le bassin de la Moselle dont les contrastes d'ordre climatique, orogénique, etc., n'ont rien de comparable à ceux du bassin du Cris. Indépendamment du critère de choix, la sélection du meilleur modèle de référence permet des résultats comparables au modèle régional du Cris (5).

\section{4 - EXTRAPOLATION DES DISTRIBUTIONS AUX GRANDES PÉRIODES DE RETOUR (10 < T(AN) $\leq 1000)$}

\subsection{Modélisation locale}

La forme d'extrapolation des distributions consolidées (2) aux quantiles de débit de grande période de retour est une forme progressive (MICHEL et OBERLIN, 1987) dérivée de la méthode du GRADEX (GUILLOT et DUBAND, 1967). Cette forme progressive (8) permet d'extrapoler les distributions pour des durée $d$ comprises entre $D / 2$ et $5 D$ (GALÉA et JIN, 1990) à l'aide des gradex de pluie observés sur le bassin : 


$$
V_{d}(T)=V_{L}(d, 10)+G p_{d} \ln \left(1+\frac{G q(d)}{G p_{d}} \cdot \frac{T-10}{10}\right)
$$

où $\quad V_{L}(d, 10)$ : est déduit de l'expression (2) ;

$G p_{d}$ : gradex des pluies de bassin de durée $d\left(\mathrm{~m}^{3} / \mathrm{s}\right)$.

Le passage aux quantiles de pointe privilégie la distribution relative à $d=D$ et un coefficient de forme moyen $\bar{r}_{s}\left(r: \frac{Q I X}{V_{d=D}}\right)$ déduit des échantillons de débit de pointe et de débit moyen sur $D$.

soit :

$$
Q I X(T)=V_{d=D}(T) \cdot r
$$

Si l'on généralise le gradex des pluies $G p_{d}$ avec la durée $d(9)$, nous obtenons le model QdF d'extrapolation local (10) qui permet d'homogénéiser et de consolider avec $d(0 \leq d \leq 5 \mathrm{D})$ les extrapolations selon (8).

$$
G p(d)=\frac{1}{x 7 \cdot d+x 8}+x 9
$$

où $x 7, x 8$ et $x 9$ sont les paramètres calés, satisfaisants à l'ensemble des quantiles de crue de durée $d$ et de période moyenne de retour $T(20 \leq T(a n)$ $\leq 1000)$

$$
V_{L}(d, T)=V_{L}(d, 10)+G p(d) \cdot \ln \left(1+\frac{G q(d)}{G p_{d}} \cdot \frac{T-10}{10}\right)
$$

Le graphe de la figure 17 donne une représentation de l'erreur selon (3) liée à la modélisation locale (10) par rapport aux quantiles extrapolés (8).

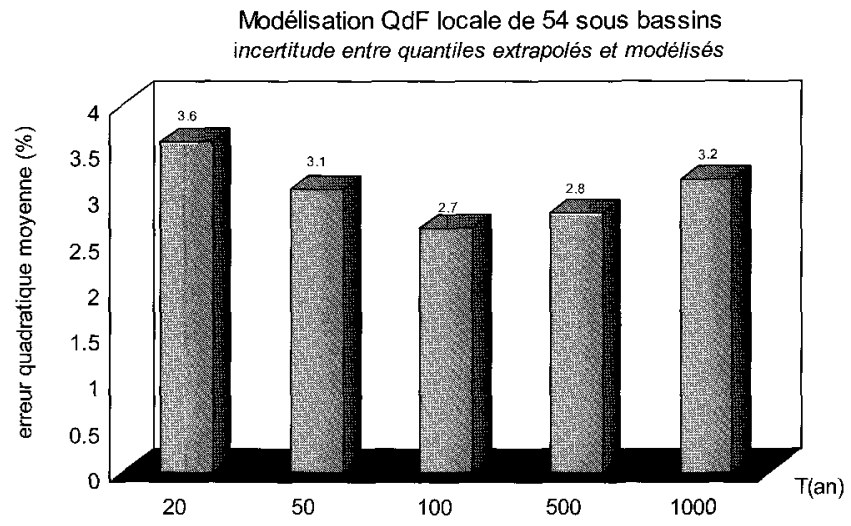

Figure 17 Incertitude liée à la modélisation locale (54 sous-bassins). Local modeling incertitude (54 sub-catchments). 


\subsection{Approche régionale classique}

Le passage à la modélisation régionale pour ce qui concerne les quantiles rares est analogue à ce qui a été présenté pour les quantiles de crue de faible période de retour. Autrement dit, il suffit de définir une fonction régionale adimensionnelle du gradex des pluies (11).

$$
G p_{R}(d / D)=\frac{1}{x 7_{R} \cdot d / D+x 8_{R}}+x 9_{R}
$$

Finalement, sont obtenus pour chaque sous bassin hydrographique et le bassin hydrographique du Cris, les valeurs des paramètres régionaux du gradex des pluies (tableau 3).

Tableau 3 Paramètres régionaux du gradex des pluies.

Table 3 Regional parameters of rainfalls gradex.

\begin{tabular}{|c|c|c|c|}
\hline Sous bassins hydrographiques & $X 7_{\boldsymbol{R}}$ & $X \mathbf{8}_{\boldsymbol{R}}$ & $\boldsymbol{X 9 _ { \boldsymbol { B } }}$ \\
\hline Crisul Alb $\left(3911 \mathrm{~km}^{2}\right)$ & 1,284 & 1,808 & 0,019 \\
Crisul Negru $\left(4495 \mathrm{~km}^{2}\right)$ & 1,310 & 1,907 & 0,021 \\
Crisul Repede $\left(5878 \mathrm{~km}^{2}\right)$ & 1,172 & 1,851 & 0,032 \\
\hline Bassin hydrographique & $\boldsymbol{X 7}_{\boldsymbol{R}}$ & $\boldsymbol{X 8 _ { \boldsymbol { R } }}$ & $\mathbf{X 9}_{\boldsymbol{R}}$ \\
\hline Cris $\left(14300 \mathrm{~km}^{2}\right)$ & 1,255 & 1,855 & 0,024 \\
\hline
\end{tabular}

Ces résultats portés sur un même graphe (figure 18) permettent de conclure à la représentativité du gradex régional du Cris.

Bassin hydrographique du Cris et ses 3 sous bassins hydrographiques Comparaison des gradex de pluie régionaux

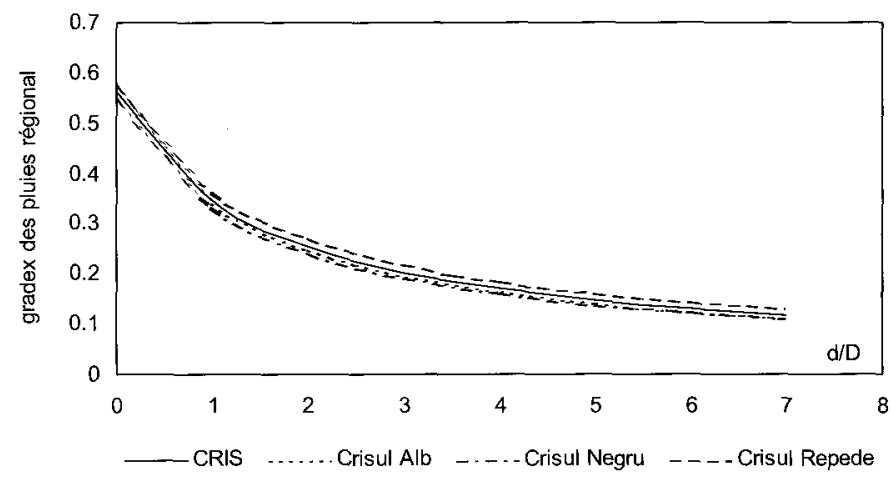

Figure 18 Comparaison des gradex de pluie régionaux.

Regional rainfall gradex comparison.

Les quantiles de crue de fréquence rare $(T>10$ ans) et pour toute durée $d$ ( 0 $\leq \mathrm{d}(\mathrm{j}) \leq 10)$, en un site cible, pourront être déduits de l'expression régionale (12): 


$$
V_{R}(d, T)=V_{R}(d, 10)+\left[G p_{R}(d) \cdot \ln \left(1+\frac{G q_{R}(d)}{G p_{R}(d)} \cdot \frac{T-10}{10}\right)\right] \cdot Q 10_{\text {site cible }}
$$

où

$$
V_{R}(d, 10) \text { : est donné par (5) }
$$

avec

$$
\begin{aligned}
& G q_{R}(d)=\frac{1}{X 1_{R} \cdot d / D_{\text {site cible }}+X 2_{R}}+X 3_{R} \\
& G p_{R}(d)=\frac{1}{X 7_{R} \cdot d / D_{\text {site cible }}+X 8_{R}}+X 9_{R}
\end{aligned}
$$

À la figure 19 nous présentons un exemple de validation (code station : 36115 ) du modèle régional du Cris (12) pour l'estimation des quantiles de crue de grande période de retour.

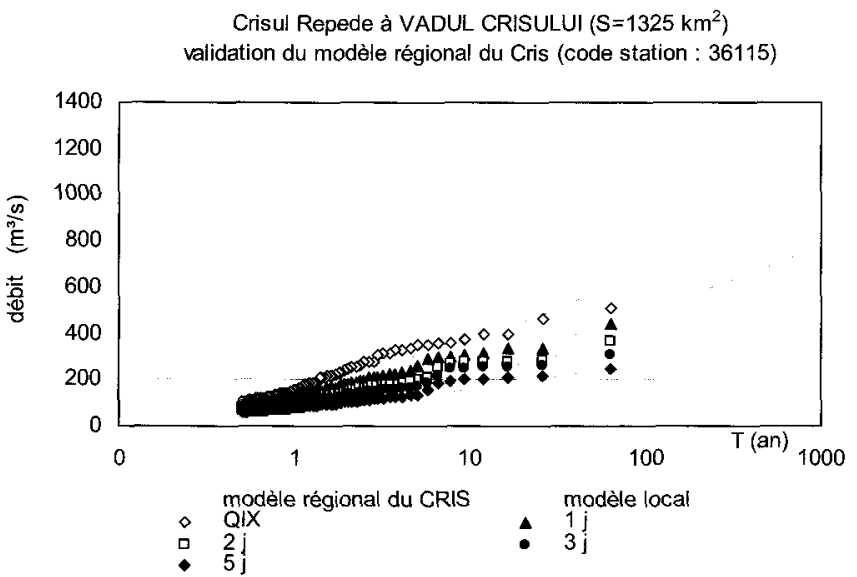

Figure 19 Exemple de validation du modèle régional du Cris (12).

Validation example of Cris regional model (12).

\subsubsection{Jeu de calage}

À la figure 20, nous présentons un graphe de comparaison des estimations par modélisation locale ou régionale ainsi qu'un graphe de synthèse de l'erreur.

\subsubsection{Jeu de validation}

Une même présentation est donnée que précédemment pour le jeu de validation (figure 21).

\subsection{Approche par modèles de référence}

Les paramètres des modèles de référence concernant le gradex adimensionnel des pluies $G p_{\text {ref }}(d / D)$ sont donnés au tableau 4 ; dans l'équation (12) $R$ sera substitué par ref. 
Bassin hydrographique du Cris : quantiles de débit centennaux jeu de calage de 54 sous bassins

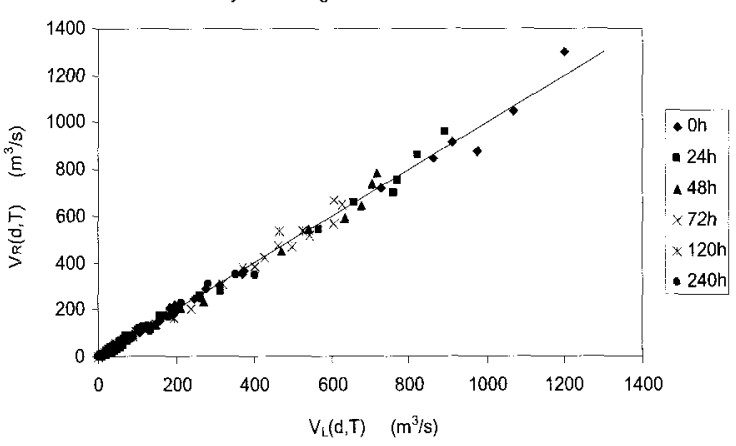

Modélisation $Q d F$ locale et régionale incertitude entre quantiles modélisés, jeu de calage de 54 sous bassins

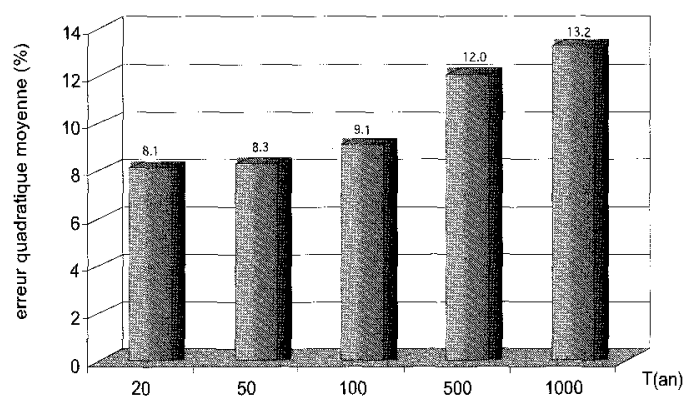

Figure 20 Modélisation locale (10) et régionale (12), comparaison des quantiles 100 ans et graphe de synthèse.

Local (10) and regional (12) modeling, 100-year quantiles comparison and synthesis graph.

Tableau 4 Valeurs des paramètres des trois modèles QdF de référence.

Table 4 Parameters values of the three QdF reference models.

\begin{tabular}{|c|c|c|c|}
\hline $\begin{array}{c}\text { Modèles QdF } \\
\text { de référence }\end{array}$ & $\mathbf{X}_{\text {ref }}$ & $\mathbf{X 8}_{\text {ref }}$ & $\mathbf{X 9}_{\text {ref }}$ \\
\hline (1) & 1,083 & 1,750 & 0,000 \\
$(2)$ & 1,560 & 1,910 & 0,085 \\
$(3)$ & 0,569 & 0,690 & 0,046 \\
\hline
\end{tabular}

Pour le jeu de validation nous présentons (figure 22) le graphe de synthèse de l'erreur concernant la modélisation régionale classique ou de référence par rapport à la modélisation locale (10).

Ces résultats relatifs aux fortes périodes de retour (figure 22) sont à comparer à ceux obtenus pour les faibles périodes de retour (figure 16).

Tout d'abord, concernant le modèle régional du Cris, on constate dans les deux cas de faibles erreurs par rapport aux observations locales. Ceci indique 


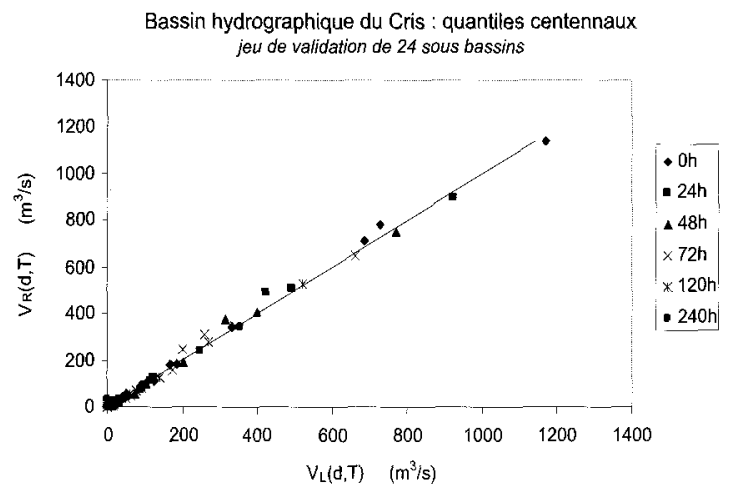

Modélisation QdF locale et régionale

incertilude entre quantiles modélisés, jeu de validation de 24 sous bassins

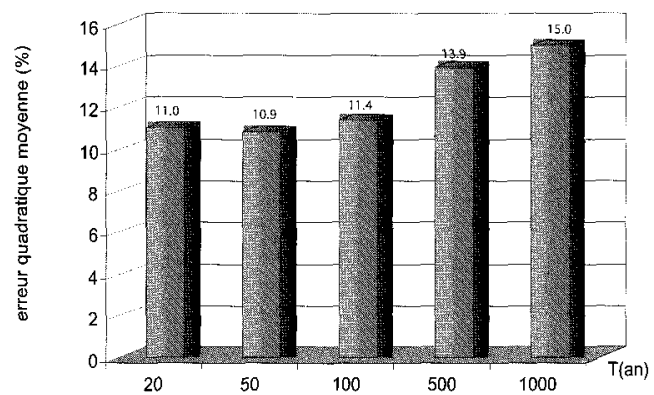

Figure 21 Validation du modèle régional, comparaison des quantiles 100 ans et graphe de synthèse.

Regional model validation, 100-year quantiles comparison and synthesis graph.

Modélisation $\mathrm{QdF}$ régionale et de référence

incertitude liée à la modélisation, jeu de validation de 24 sous bassins

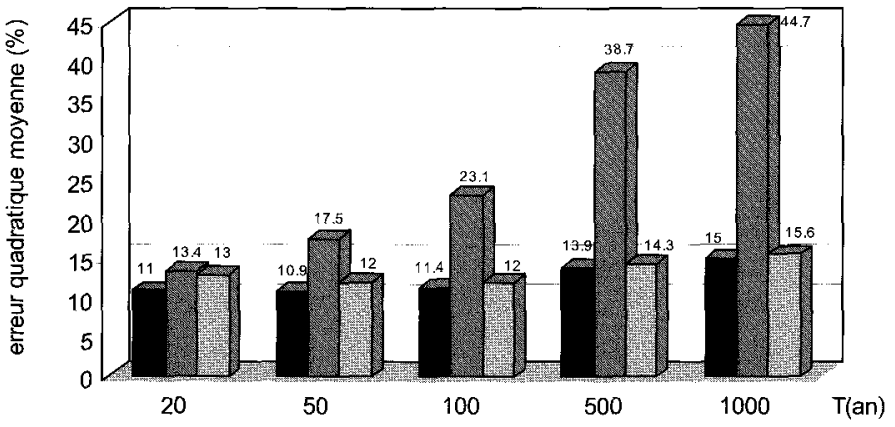

modèle QdF régional du Cris (12)

. modèle QdF de référence (le meilleur des 3 selon (7))

- modèle QdF de référence (selon le critère de choix)

Figure 22 Comparaison des erreurs quadratiques moyennes des modèles QdF. Comparison of QdF models mean quadratic errors. 
que l'ensemble de cette région a une bonne homogénéité hydrologique, aussi bien pour les distributions de débit "observées " (faibles périodes de retours) que pour les gradex de pluie (extrapolations aux fortes périodes de retour).

Concernant l'approche par modèle de référence on constate ici une grande différence dans les résultats, par rapport à la figure 16. Cette fois-ci, le modèle de référence désigné par le critère de choix donne de faibles erreurs, similaires à celle du modèle régional du Cris. En revanche, le meilleur des trois modèles de référence désigné pour les faibles périodes de retour (figure 16) conduit quant à lui à de fortes erreurs lorsqu'on l'applique aux périodes de retour rares (figure 22).

Ces résultats s'expliquent par la méthode d'extrapolation aux fréquences rares utilisée. Celle ci est liée à la méthode du GRADEX, faisant intervenir les gradex de pluie du bassin étudié. Utilisant ces mêmes gradex de pluie, le critère de choix décrit figure 15 revient donc à choisir le modèle de référence dont les extrapolations sont les plus compatibles avec celles de la méthode du GRADEX, et cela, sans tenir compte des résultats sur les faibles périodes de retour. De ce fait, les erreurs observées pour les faibles périodes de retour se retrouvent « corrigées " lorsqu'on s'intéresse aux fortes périodes de retour.

\section{5 - CONCLUSION}

Cette étude a comparé les résultats de l'approche modèles de référence et ceux d'une régionalisation classique des courbes QdF. Comme nous l'avons $\mathrm{vu}$, dans les deux cas, des courbes QdF adimensionnelles sont dénormées par deux descripteurs du site cible : le débit décennal Q10 et une durée caractéristique de crue $D$. La différence entre les deux approches réside dans l'ajustement de ces courbes adimensionnelles. Pour les modèles de référence, trois jeux de courbes QdF adimensionnelles sont disponibles, chacun ayant été ajusté une fois pour toutes sur un bassin français représentatif d'un régime hydrologique particulier. Le choix du jeu de courbes à dénormer localement s'effectue par un critère impliquant les gradex de pluie observés sur le site cible, ainsi que les paramètres locaux Q10 et $D$. Dans le cas de la régionalisation classique, un seul jeu de courbes QdF adimensionnelles est ajusté sur l'ensemble d'une région hydrologique supposée homogène.

Les résultats présentés montrent une bonne validité du modèle régional ajusté sur l'ensemble du Cris. Ceci indique que la zone étudiée est relativement bien homogène. Concernant les modèles de référence, leur critère de choix n'apparaît pas pertinent lorsqu'on s'intéresse aux faibles périodes de retour, mais se révèle significatif pour les fortes périodes de retour. Ce résultat est en grande partie dû à la méthode d'extrapolation appliquée. Celle ci est liée à la méthode du GRADEX (GUILLOT et DUBAND, 1967) et utilise l'information locale sur les gradex de pluie, comme cela est souvent le cas en France.

II est à noter que ces modèles de référence établis sur des chroniques de débit et pluie d'avant 1992 n'ont pas été réactualisés. L'exemple du bassin du Cris montre qu'ils n'en gardent pas moins un caractère opérationnel. 


\section{RÉFÉRENCES BIBLIOGRAPHIQUES}

CTGREF, 1980. Synthèse nationale sur les crues des petits bassins, fascicule 2 : la méthode SOCOSE, Informations Techniques $n^{\circ} 38-2$ (juin 1980).

COX D.R., 1962. Renewal Theory. Methuen \& Co, LTD, London, $186 \mathrm{p}$.

DALRYMPLE T., 1960. Flood frequency analysis. U.S. Geol. Surv. Water Supply Pap., 1543A.

FELLER W., 1966. An Introduction to Probability Theory and its Applications. John Wiley \& Sons, Inc., New York, 2, 346372.

GALÉA G., JIN L., 1990. Modèles descriptifs synthétiques des connaissances régionales en crue ; représentativité spatiale et domaine de validité. CemagrefLyon/DEA ULPS/ENITRTS.

GALÉA G., PRUDHOMME C., 1997a. Notions de base et concepts utiles pour la compréhension de la modélisation synthétique des régimes de crue des bassins versants au sens des modèles $Q d F$. Revue des Sciences de l'Eau, 1, 83-101.

GALÉA G., PRUDHOMME C., 1997b. Synthèses régionales des régimes hydrologiques : approche QdF et régionalisation. Cemagref Éditions, Antony, in: FRIEND projects $\mathrm{H}-5-5$ and 1.1 Third report: 1994-1997, 1997, 99-108.

GALÉA G., GENDREAU N., JAVELLE P., POINARD D., PUECH C., SCHNEIDER M., 2001. Régimes des crues des sousbassins de la Moselle française/modèles régionaux et SIG associé. Projet PACTES, UR Hydrologie-Hydraulique, Cemagref-Lyon, octobre 2001.

GUILLOT P., DUBAND D., 1967. La méthode du GRADEX pour le calcul de la probabilité des crues à partir des pluies. Journées de la SHF (septembre), question 1 , rapport 7 , Paris.

GREHYS, 1996a. Presentation and review of some methods for regional flood frequency analysis. Journal of Hydrology, 186, 63-84.

GREHYS, (1996b. Inter-comparaison of regional flood frequency proceedures for
Canadian rivers, Journal of Hydrology, 186, 85-103.

JAVELLE P., 1997-2001. Caractérisation du régime des crues : le modèle débitdurée-fréquence convergent - Approche locale et régionale. UR HydrologieHydraulique, Cemagref, groupement de Lyon, thèse soutenue le 01 mars 2001 à I'INPG (MMGE).

JAVELLE P., GRÉSILLON J.M., GALÉA G., 1999. Modélisation des courbes Débitdurée-Fréquence en crues et invariance d'échelle. Comptes Rendus de l'Académie des Sciences. Paris, Sciences de la terre et des planètes, 329, 39-44.

JAVELLE P., GALÉA G., GRÉSILLON J.M., 2000. L'approche débit-durée-fréquence: historique et avancées, Revue des Sciences de l'Eau, 13 (3), 303-321.

JAVELLE P., OUARDA T., LANG M., BOBÉE B., GALÉA G., GRÉSILLON J.M., 2002. Development of regional flood-durationfrequency curves based on the indexflood method. Journal of Hydrology, 258 (1-4), 249-259.

LANG M., OUARDA T., BOBÉE B., 1999. Towards operational guidelines for overthreshold modeling. Journal of Hydrology, 225, 103-117.

MICHEL. C., OBERLIN G., 1987. Seuil d'application de la méthode du GRADEX $L a$ Houille Blanche $n^{\circ} 3,199-203$.

MIC R., 1997-2002. Régionalisation des événements hydrologiques extrêmes. Thèse soutenue à l'INMH de Bucarest (Roumanie, le 22 février 2002).

OUARDA T., LANG M., BOBÉE B., BERNIER J., BOIS P., 1999. Synthèse de modèles régionaux d'estimation de crue utilisés en France et au Québec. Revue des Sciences de l'Eau, 12 (1), 155-182.

PRUDHOMME C., 1992-1995. Modèles synthétiques des connaissances en hydrologie. Application à la régionalisation des crues en Europe alpine et méditerranéenne. Thèse de Doctorat en Hydrologie soutenue le 8 décembre 1995. Cemagref-Lyon, Division HydrologieHydraulique, LHM Montpellier II. 